\title{
Electron Microscopy Used for the Selection of the Microbial Strains Requested in the Case of Hydrolyzed Wood Wastes Conversion to Fodder Yeasts
}

\section{Ana- Despina Ionescu*, Angela Căşărică and Nicoleta Ene}

National Institute for Chemical- Pharmaceutical Research and Development Bucharest, Vitan Avenue, Bucharest, Romania

*Corresponding Author: Ana-Despina Ionescu, National Institute for ChemicalPharmaceutical Research and Development - Bucharest, Vitan Avenue, Bucharest, Romania.
Received: September 17, 2020

Published: October 30, 2020

(C) All rights are reserved by Ana- Despina Ionescu., et al.

\section{Abstract}

According to the literature data, the conversion of the ligno-cellulosic materials towards fodder yeasts involves first the use of some selected microbial strains able to produce the enzymatic factors and then the optimal yeasts. The factors with influence on this bioprocess are obviously the applied parameters but also the morphology of the studied microbial strains.

The optimal used parameters can be establish by analytical determinations, but the morphology must be observed by optical microscopy, electron microscopy, X-ray photoelectron spectroscopy, infrared spectroscopy, contact angle, electrophoretic mobility measurements or even by atomic force microscopy. Our studies were realized by electron microscopy on the some previously selected strains of filamentous fungi and yeasts.

Keywords: Electron Microscopy; Wood; Cellulases; Yeasts

\section{Abbreviations}

AN-8: Abbreviation for a selected fungal strain of Aspergillus niger; AN-3: Abbreviation for a selected fungal strain of Aspergillus niger; CB- 909: Abbreviation for a selected strain of Candida robusta

\section{Introduction}

According to the literature data, the conversion of the lignocellulosic materials towards fodder yeasts involves first the use of some selected microbial strains able to produce the enzymatic factors and then the optimal yeasts. The factors with influence on this bioprocess are obviously the applied parameters but also the morphology of the studied microbial strains.

The optimal used parameters can be establish by analytical determinations, but the morphology must be observed by optical microscopy, electron microscopy, X-ray photoelectron spectroscopy, infrared spectroscopy, contact angle, electrophoretic mobility measurements or even by atomic force microscopy. [1-11,16].
As we can understand from these numerous literature references, because of the small cell sizes, subcellular structures have long been difficult to visualize using diffraction-limited light microscopy [2].

Due to this situation, the microscopes with higher performances were used during the last year, in order to probe intracellular and extracellular structures with unprecedented resolutions, enabling researchers to study their organization, dynamics and interactions in individual cells, at the single-molecule level [2].

Moreover, we can find the most recent articles indicating the advantages of the electron microscopy [12-15,17].

All these verified documents can indicate that the scanning Electron Microscopes (SEMs) are used across a number of industrial, commercial and research applications. From cutting edge fabrication processes to forensic applications, there's a diverse range of practical applications [17]. 
Our researches were carried out on the different selected strain of the filamentous fungi Aspergillus niger (know as a good producer of the cellulase complex) preserved on a solid media and also on some selected yeast strains used with good results in order to obtain fodder yeast biomass.

The results obtained by analytical determinations concerning the level of their products were coordinated with the morphological aspects observed by electron microscopy.

\section{Materials and Methods}

The studied fungal strains were preserved on a sterilized solid media containing: boiled potatoes, dextrose and agar-agar (pH 7,1 - 7,2). The same fungal strains can be kept also on solid media containing malt extract and agar- agar or by a shacked liquid culture based on soybean meal and ground corn cobs.

In order to establish the correlation between the morphological structure and the physiological functions, the electron microscope was used on 3 different selected strains of Aspergillus niger, by observing the appeared differences between their cellular ornamentations, such as: crests, thorns, etc. (differences between their size, shape or number).

After the use of those fungal strains producing of an enzymatic complex and performing the hydrolysis step, the process for obtaining the fodder yeasts was continued by the culture of different yeast strains such as Candida arborea, Candida tropicalis, Candida utilis, Candida robusta and Candida scotii. The yeast strains were preserved on solid media containing: D-glucose, peptone and agaragar ( $\mathrm{pH} \mathrm{6,0)} \mathrm{and} \mathrm{the} \mathrm{sample} \mathrm{preparation} \mathrm{was} \mathrm{realized} \mathrm{according}$ to the standardized procedures indicated for an electron microscope type Philips.

\section{Results and Discussions}

This paper presents 3 examples of photos realized by using the electron microscope in order to discover some possible correlations between the fine morphological appearance of the applied microbial strains and their obtained performance in the case of conversion of the wood hydrolyzed wastes towards fodder yeasts.

This images are presented by the figure 1-3.

The indications obtained by using the electron microscopy techniques have demonstrated that there are some morphological differences between the previously isolated microbial strains (selected by cultures on solid or liquid media), for example the presence of some crests, thorns (cellular ornamentation), the differences concerning the cell wall thickness, etc.

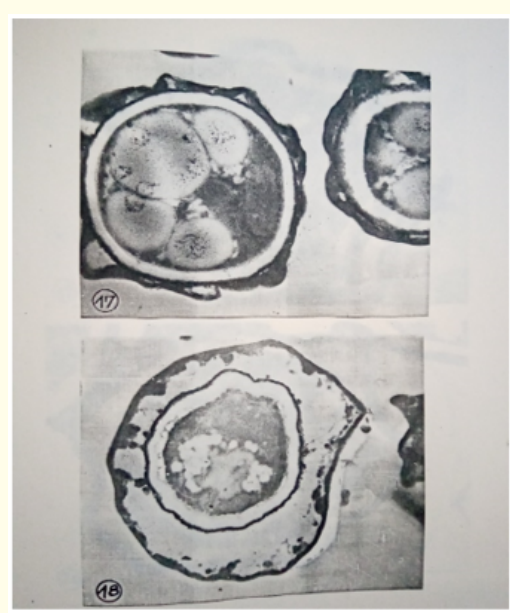

Figure 1: The cellular ultrastructure in the case of ornamentation at the fungal strain AN-8, observed by electron microscopy.

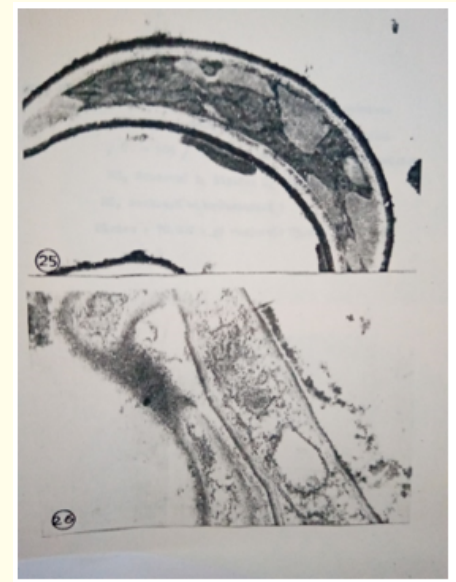

Figure 2: The cellular ultrastructure in the case of mold hyphae from the fungal strain AN-3.

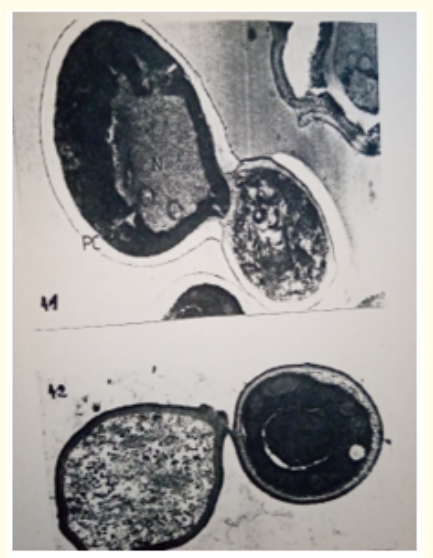

Figure 3: The cellular ultrastructure and the cell division in the case of the yeast strain CB-909. 


\section{Conclusion}

- This paper presents some aspects concerning the methods which can be used for the selection of the best microbial strains requested for the conversion of the cellulosic wastes towards fodder yeasts.

- The indications obtained by using the electron microscopy techniques have demonstrated that there are some morphological differences between the previously isolated microbial strains (selected by cultures on solid or liquid media), for example the presence of some crests, thorns (cellular ornamentation), the differences concerning the cell wall thickness, etc.

- For monitoring the current evolution of this kind of processes, the optimal methods are represented by the analytical determinations, whereas the electron microscopy looks to be a quite expensive solution and asking additional specific work conditions, usually difficult to obtain.

\section{Bibliography}

1. Yves F Dufrene. "Atomic Force Microscopy, a Powerful Tool in Microbiology". Journal of Bacteriology 184.19 (2002).

2. Jie Xiaol and Yves F Dufrene. "Optical and force nanoscopy in microbiology". Nature Microbiology 1.11 (2016).

3. Michael Elbaum. "Quantitative Cryo-Scanning Transmission Electron Microscopy of Biological Materials". Advanced Materials 30.41 (2018).

4. Alioscha-Perez M., et al. "A Robust Actin Filaments Image Analysis Framework". PLOS Computational Biology 12.8 (2016).

5. Flavien Pillet., et al. "Uncovering by atomic force microscopy of an original circular structure at the yeast cell surface in response to heat shock". BMC Biology 6 (2014).

6. Jean Marie Francois., et al. "Use of atomic force microscopy (AFM) to explore cell wall properties and response to stress in the yeast Saccharomyces cerevisiae". Current Genetics 59.4 (2013): 187-196.

7. Marion Schiavone., et al. "Evidence for a Role for the Plasma Membrane in the Nanomechanical Properties of the Cell Wall as Revealed by an Atomic Force Microscopy Study of the Response of Saccharomyces cerevisiae to Ethanol Stress". Applied and Environmental Microbiology 82.15 (2016).

8. Etienne Dague., et al. "Towards a nanoscale view of fungal surfaces". Yeast 24.4 (2007): 229-237.
9. Vincent Dupres., et al. "Microbial nanoscopy: a closer look at microbial cell surfaces". Trends in Microbiology 18.9 (2010): 395-405.

10. Peter Hinterdorfer., et al. "Single-molecule imaging of cell surfaces using near-field nanoscopy". Accounts of Chemical Research 45.3 (2012): 327-336.

11. Daniel J Muller and Yves F Dufrene. "Atomic force microscopy: a nanoscopic window on the cell surface". Trends in Cell Biology 21.8 (2011): 461-469.

12. Yves F Dufrene. "Atomic force microscopy of fungal cell walls: an update". Yeast 27.8 (2010): 465-471.

13. Christine G Golding and Lindsey L Lamboo. "The scanning electron microscope in microbiology and diagnosis of infectious disease". Nature Scientific Reports (2016).

14. Schiavone M., et al. "AFM dendritips functionalized with molecular probes specific to cell wall polysaccharides as a tool to investigate cell surface structure and organization". Cell Surfer 5 (2019): 100027.

15. Cristina Martinez- Torres., et al. "Revealing the assembly of filamentous proteins with scanning transmission electron microscopy". PLoS One 14.12 (2019).

16. Goossens KV., et al. "Molecular mechanism of flocculation selfrecognition in yeast and its role in mating and survival". mBio 6.2 (2015).

17. ATA SCIENTIFIC. "The Applications and Practical Uses of Scanning Electron Microscopes". ATA SCIENTIFIC Instruments (2019).

\section{Assets from publication with us}

- Prompt Acknowledgement after receiving the article

- Thorough Double blinded peer review

- Rapid Publication

- Issue of Publication Certificate

- High visibility of your Published work

Website: https://www.actascientific.com/

Submit Article: https://www.actascientific.com/submission.php

Email us: editor@actascientific.com

Contact us: +919182824667 